\title{
Theoretical color-magnitude diagrams and the star forming histories of interacting open multi-population model galaxies: bursts and busts
}

\author{
G. Valle ${ }^{1}$, M. Cignoni ${ }^{1}$, and S. N. Shore ${ }^{1,2}$ \\ 1 Dipartimento di Fisica "Enrico Fermi”, Università di Pisa, Largo Pontecorvo 3, 56127 Pisa, Italy \\ 2 INFN - Sezione di Pisa, Largo Pontecorvo 3, 56127 Pisa, Italy \\ e-mail: shore@df.unipi.it
}

Received 4 April 2005 / Accepted 13 June 2005

\begin{abstract}
This note presents theoretical color-magnitude diagrams (CMDs) and metallicity evolution for Galactic multipopulation models coupled to stellar evolution models for systems undergoing stripping and re-accretion of ambient material, called "open" systems in our recent studies (Valle et al. 2005, A\&A, 435, 551). We show that the observables for such systems, in particular those related to the recovered star formation as a function of time, are ambiguous - systems with non-monotonic star formation rates can appear as either bursting systems or galaxies with a hiatus in the star formation.
\end{abstract}

Key words. galaxies: evolution - galaxies: interactions

\section{Introduction}

This research note extends the work of Valle et al. (2005, hereafter VSG05) concerning the effects of environment on the evolution of galactic populations and metallicities. Our intent here is to underline some observational consequences of this approach. To place our simulations in context, we first summarize some basic points already extensively treated in our previous work. Our standard model of Galactic chemical evolution uses a multi-zone, multi-population approach (cf. Ferrini et al. 1992; Shore \& Ferrini 1995; Valle et al. 2002). The system is schematicized using three distinct zones - the halo (HA) (in our models this implicitly includes the bulge and spheroid), thick disk (TD), and thin disk (DI) - that exchange mass, each consisting of three interconverting phases: diffuse gas (g), clouds, (c), and stars (s). We use a coupled population dynamical approach which, although without explicit thermomechanical prescriptions for material transport or chemodynamical feedback, provides the star formation rate as a function of time directly from the model equations and all chemical evolution is followed without instantaneous recycling.

In VSG05 we used this approach to study stochastic accretion and mass loss within closed and open systems but concentrated on the star formation rates and chemical evolution. However, because we have self-consistent results for both, we can link them to produce population synthetic color-magnitude diagrams (CMDs) and other observable diagnostics of galactic evolution. One of the most interesting results to emerge from
VSG05 was, in our view, the phenomenon of a burst in reverse star formation - that following a collision, subsequent replenishment of the interstellar medium through mass shedding by evolving stars would restart star formation after a considerable delay, producing the appearance of a bursty star forming history. This was notable since there is no provision taken in the models for enhanced induced star formation during either the collisional or stripping/refilling events. This also, under extreme conditions, led to galaxies with inverted metallicity-age relations, younger populations that can have lower metallicity than more evolved components depending on the course of the re-accretion process, a phenomenon particularly relevant for galaxies in clusters. For this reason, we follow up in this paper only simulations of collisions and collision/stripping/reaccretion scenarios and compare these to the standard case. We updated the model by including the Kroupa initial mass function (IMF) (Kroupa 2002), replacing that obtained from analysis of molecular cloud fragmentation used in all previous papers in this series (i.e. Ferrini et al. 1992). All process rates and chemical yields have been recomputed using the newly adopted IMF to be consistent with the stellar evolution simulations.

\section{Population synthesis code}

The population simulations use the Pisa evolutionary library (e.g. Cariulo et al. 2004). The evolutionary tracks used in the simulations were computed assuming a primordial helium 
abundance $Y_{\mathrm{P}}=0.23$ and a fixed enrichment ratio $\Delta Y / \Delta Z=2.5$ (see Pagel \& Portinari 1998; Castellani et al. 1999). In part because of the schematic nature of the models we present here, we have not included a binary population. We have not varied the IMF in view of the uncertainties regarding its evolution. For the same reason, we have not included a binary population since we still lack detailed knowledge of the statistical distribution of the mass ratio $q$ and even the fraction of binaries present in the solar neighborhood. Because the evolution of close binaries also depends on the initial period distributions, the range of phenomenology is far wider than even for passive (widely separated independently evolving) components. In addition, because the mass ratios are drawn from what must be a continuous distribution, binaries will smear the width of the giant branch, and main sequence, without necessarily producing substructure (see Hurley \& Tout 1998); in principle, this displacement can introduce a gap in the RGB region, but it is very improbable that all binaries have a companion of equal mass. Although the Monte Carlo procedure we use here is designed to model the Solar neighborhood based on the Hipparcos measurements, which is dominated by small samples, the same methods can be used to model a galaxy assuming as inputs the star formation rate and metallicity-age relation. There is no gradient in the metallicity, no structure, and all parameters for the stellar population are assumed to be independent of position. Thus, we emphasize the schematic nature of this calculation as we did in VSG05 for the star-forming and abundance histories - and although we model the full galactic evolution we include only a one zone calculation (thin disk history) for the CMDs.

An essential difference between our approach to the simulated field CMDs and others in the literature has to do with the simultaneous solutions for the metal abundances and the star formation through the model equations. Any population model depends on two inputs: the age-metallicity relation and the star formation rate (for fixed IMF) as a function of time. In principle, any chemical evolution code produces a consistent history for the abundances once an assumed star formation rate is explicitly supplied. The systematic bias introduced by a specific functional choice for the SFR cannot be compensated by simply adjusting parameters. Instead, different histories are usually tried and the results compared with observations. Since we have a model system for the evolution equations, at least the systematic bias can be reduced - although not eliminated - because a large variety of interlocking processes are treated simultaneously.

The simulations were performed using the Monte Carlo method previously described by Castellani et al. (2002) and Cignoni et al. (2003); no mass loss is used for the RGB so the horizontal branch is more prominent in the simulated CMDs than in real galaxies. It consists of sampling a library of metallicity dependent isochrones with a constant initial mass function according to the chemical history. The full nonlinear galactic model produces a (numerical) time dependent star formation rate, $\psi(t)$. The number of stars formed before some time $t_{i}, N\left(t \leq t_{i}\right)$, is equated to a random number and the distribution of times $\left\{t_{i}\right\}$ is then used to sample the evolutionary tracks with the appropriate metallicity $Z\left(t_{i}\right)$. The masses are
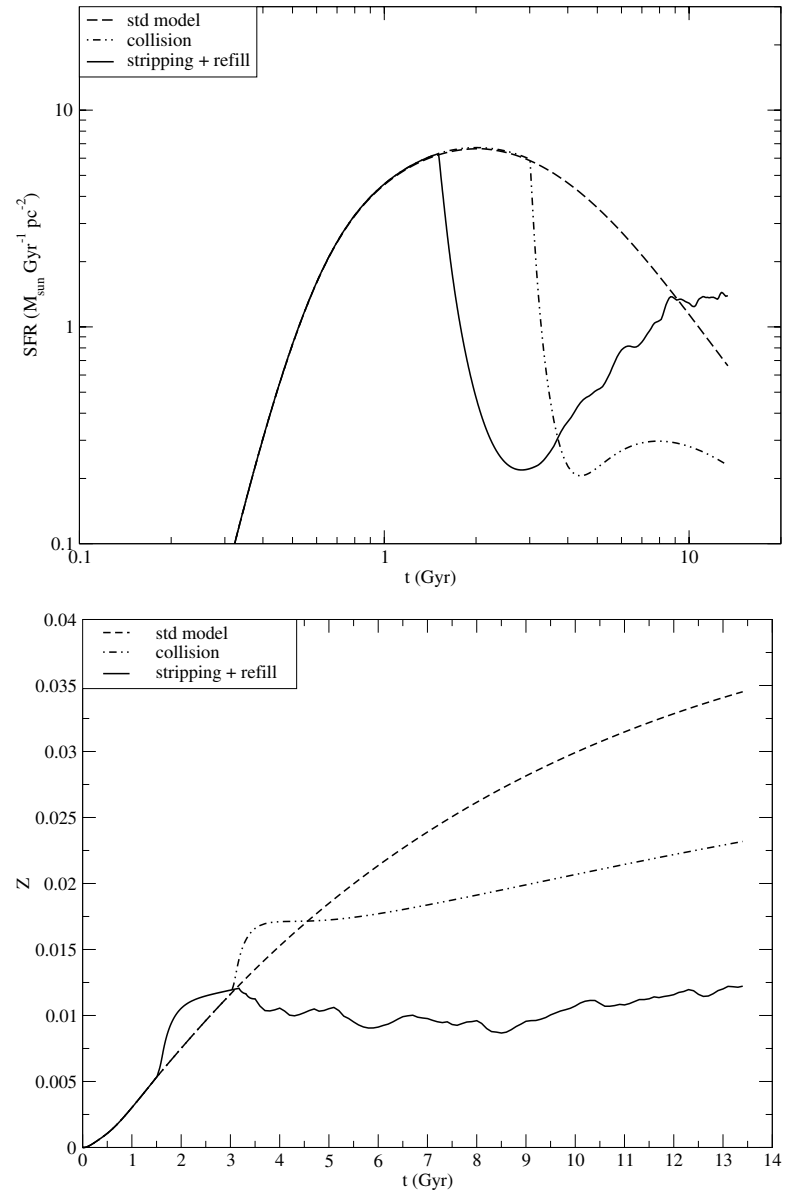

Fig. 1. a) Star formation rate (top) and b) metallicity evolution for the three scenarios (bottom). Dot-dash: collision simulation with $t_{\text {start }}=$ $3 \mathrm{Gyr}$ and $\Delta t_{\text {coll }}=130 \mathrm{Myr}$. Mass loss profiles were as in Quilis et al. (2000). Black continuous: combined effects of collision and environmental infall. The collision event was assumed to have $t_{\text {start }}=1.5 \mathrm{Gyr}$ and $\Delta t_{\text {coll }}=130 \mathrm{Myr}$. After the collision, the model assumes stochastic infall and re-accretion of primordial material starting at $t=3 \mathrm{Gyr}$. About $40 \%$ of the total initial mass was removed during the collision and then about $25 \%$ was restored during the subsequent re-accretion. The comparison with the standard model case is also shown (dash). Note: in this figure we display only the thin disk evolution and update the VSG05 IMF (see text).

distributed according to an assumed time-invariant initial mass function, in this case the same one used for the galactic evolutionary model. Each track is selected based on the agemetallicity relation for the system derived from the star formation model, $Z(t)$, and to keep as close as possible to observables, we used the color transformations from Castelli et al. (1997) that are also used in the standard version of the solar neighborhood population simulation (e.g. Castellani et al. 2002; Cignoni et al. 2003).

\section{Simulation of observables}

As in our previous paper, the collisions were simulated as impulsive events starting at some time $t_{\text {start }}$ and lasting for $\Delta t_{\text {coll }}$ during which time the diffuse gas fraction is set continuously to some reduced value, including complete removal. In Fig. 1 (dot-dash) we present the case of a single collision with a 

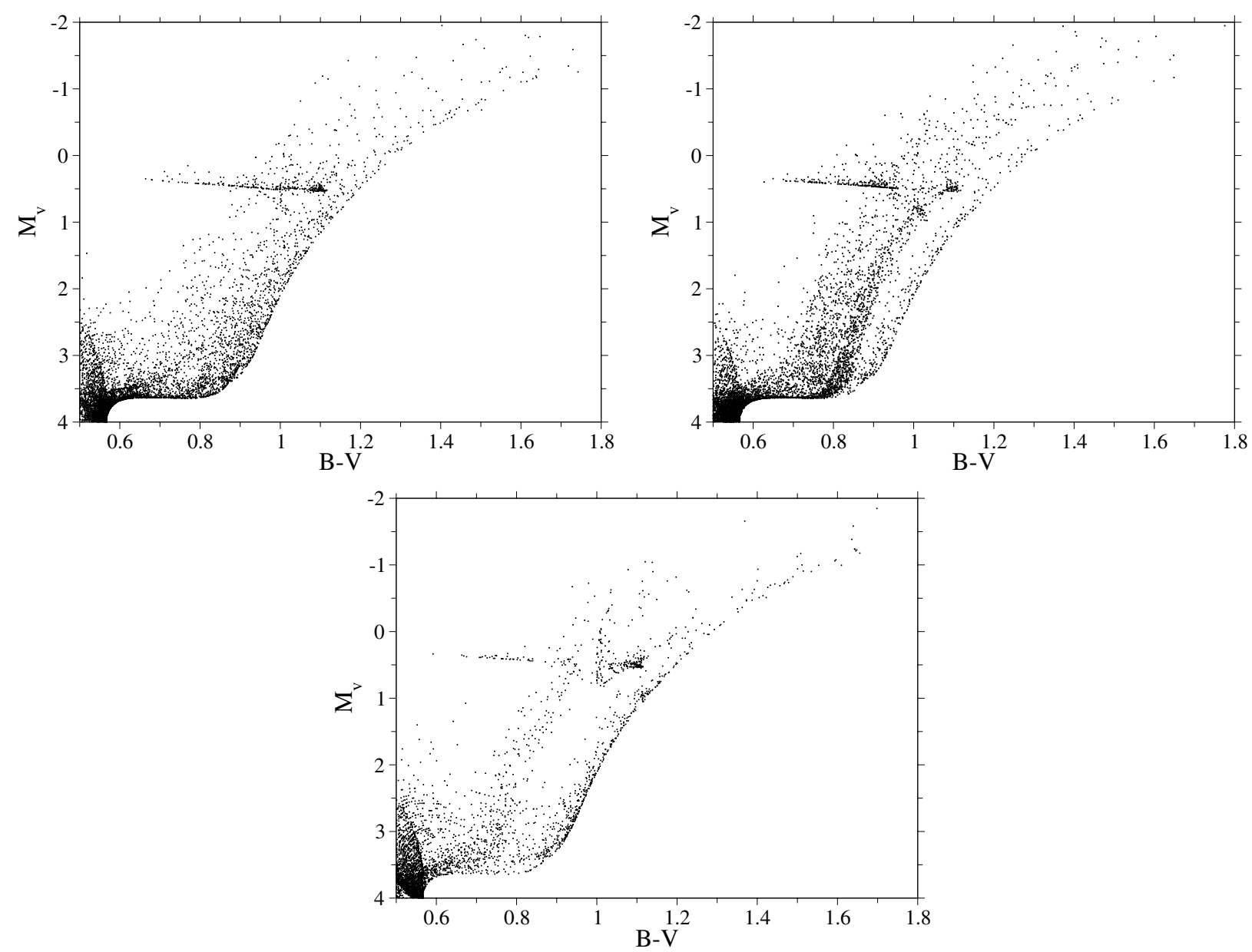

Fig. 2. Synthetic CM diagram for the cases shown in Fig. 1: a) in the standard case (top), b) in the collision case (middle) and c) the combined collision-re-accretion scenario (bottom). The color-gap in the RGB indicates a discontinuous change (jump) in the metallicity (e.g. Fig. 1b, collision case without refilling). With refilling, the metallicity produces a broader spread in the two, well-separated, RGBs with substructure reflecting the dilution due to re-accretion. For dSph and Irr, the re-accretion may not occur.

duration of 130 Myr started arbitrarily at 3 Gyr, using a mass loss history within the stripping during the collision obtained numerically by Quilis et al. (2000). This stripping history removes all diffuse gas within the galaxy, including that returned by stellar mass loss, without affecting the molecular clouds directly. The decrease in the cloud phase is entirely in response to cloud destruction and continuing star formation. We have already tested that the models are quite insensitive to the detailed history of the event but depend strongly on the efficiency of the gas removal in the initial stage of the collision and on the timing of the collision (VSG05). In (Fig. 1a, dot-dash) ${ }^{1}$, the SFR drops after the removal of the diffuse gas on the cloud destruction timescale. The replenishment of gas occurs only through processes related to stellar phase, hence slowly: mass shed by stars that have evolved within the various zones. No additional mass loss occurs from the system and the resupply timescale is determined by stellar evolution and the assumed IMF. During the collision, the metallicity initially rises by about $20 \%$ over a

\footnotetext{
1 The figures show only the evolution of the thin disk component of these systems. Even for the closed standard model, after about $1 \mathrm{Gyr}$, all halo star formation has ceased, that in the thick disk is substantially reduced, and the thin disk is the only active zone of the galaxy and traces the star formation.
}

very short time, about $\Delta t_{\text {coll }}$. Thereafter, the crash in the SFR halts metallicity production for some time, until stellar evolution resupplies disk gas and heavy elements (Fig. 1b, dot-dash).

An important feature for modeling low mass galaxies, in particular, is that because of the loss of the active phase (the molecular gas) the SFR after the collision never fully recovers its previous levels or those of the standard model. Thus, the remnant galaxy (system) permanently stays metal poor. As we will discuss in the next section, this behavior is reminiscent of the metallicity and star forming histories of dwarf galaxies in clusters that undergo very early tidal interactions while still forming stars. Notice that in the CMD (Fig. 2b), the giant branch displays a gap with the oldest population but also a broad distribution with a mean age now less than that of the system. Since we specifically ignore dynamical mixing within the galaxy and treat the system as a set of coupled zones, the disk exchanges of matter with the other zones vertically but not radially.

The final simulation assumes that both collisional stripping and accretion of ambient gas can occur. We showed a wide range of behaviors for such models in VSG05 following Vollmer et al. (2004), who point out the possible role of re-accretion of stripped gas on the evolution of cluster galaxies. 
Figures 1a and $b$ (continuous line) show the combined effects of collision and environmental infall of primordial material. The dominant effect comes from the removal of gas unless the infall rate is extremely high. Accretion doesn't simply dilute the abundances (Casuso \& Beckman 2004); we find instead that depending on the metallicity of the accreted material and the timing and rates of the stripping and filling events - the new gas powers further star formation (VSG05). The CMD resulting from this scenario is shown in Fig. 2c. Notice now the evident separation between the two RGB resulting from the cessation of star formation at about $2 \mathrm{Gyr}$.

\section{Discussion}

Multi-population models without dynamics are essentially local and the results for a larger system represent spatially isolated evolution. But there is a simpler type of galaxy for which the model may be more appropriate: dwarf systems (e.g. Pilyugin \& Ferrini 2000). Dwarf galaxies, particularly because they lack global patterns such as density waves, have become the laboratory of choice for studying the stochastic side of large scale star formation (Mateo 1998; Grebel 2004). Studies of dwarf spheroidals in the Local Group frequently find complex star forming histories. For instance, Tolstoy et al. (2003) summarize the range of behaviors for four dSph systems. For Sculptor, they derive an early peak (between 10 and $15 \mathrm{Gyr}$ ago). For Fornax and Leo I, in contrast, they find a peak of activity in the more recent interval, 1 to $8 \mathrm{Gyr}$, and for Carina they find multiple episodes - interpreted as bursts - throughout the period less than $10 \mathrm{Gyr}$ ago. In all cases, however, these galaxies are now more or less inert. The metallicity of each system is significantly below the Galactic disk value, even below the thick disk. Similar conclusions were reached by Pritzl et al. (2003) for the dwarf galaxy HIPASS 01321-37 for which again a peak in the star formation was found more recently than 10 Gyr. A recent paper by Tolstoy et al. (2004) reports evidence for multiple star forming events in Sculptor. These, occurring in its early stages (about $10 \mathrm{Gyr}$ ago) are qualitatively described as a boom-bust behavior, a series of bursts separated by a hiatus of star formation. The authors propose two broad alternative scenarios as working hypotheses: one in which the star formation is self-suppressed for some time after an initial burst, the other in which interactions with the environment - including other galaxies - trigger multiple star forming events and/or produces a cessation of the star formation for some extended time. Both behaviors are found in our models, but we need to be explicit about the qualitative comparison of behaviors. We use a galactic structure including multiple zones. At least for Irrs, there is a large scale structure (halo) including an extended envelope of gas that we model using a thick disk (which is the main contributor to the evolution of the system $)^{2}$.

\footnotetext{
${ }^{2}$ It also appears possible that the recently studied double red giant branch in $\omega$ Cen may be the result of such environmental action and further hints that this cluster may be the remnant of a dwarf galaxy (e.g. Sollima et al. 2005), but at this point we do not want to claim too much for our models.
}

\section{Conclusions}

Comparing the resulting CMDs for interacting model systems with those found in the literature, particularly for $\mathrm{dSphs,} \mathrm{we}$ propose that the assumption of a burst may not be unique; thus the number of stars formed in any moment and with any specific metallicity cannot be formulated in terms of two simple, independent, global functions $\psi$ and $Z(t)$. Any non-monotonic time history - for instance a cycling, a cessation, or an increase in the instantaneous star formation rate - may produce the same basic result in a coupled system of the kind we've used. Even the chemical evolution alone doesn't remove this ambiguity since the metallicity is essentially a cumulant of the history of star formation.

Acknowledgements. We thank the (anonymous) referee for a very supportive and helpful report, and John Beckman, Scilla Degl'Innocenti, Pepe Franco, Joachim Köppen, Daniele Galli, Pier Giorgio Prada Moroni, Jesper Sommer-Larsen, and Eline Tolstoy for preprints, discussions, and correspondence. This work was supported by MIUR.

\section{References}

Cariulo, P., Degl'Innocenti, S., \& Castellani, V. 2004, A\&A, 421, 1121 Castellani, V., Degl'Innocenti, S., \& Marconi, M. 1999, MNRAS, 303, 265

Castellani, V., Cignoni, M., Degl'Innocenti, S., et al. 2002, MNRAS, 334,69

Castelli, F., Gratton, R. G., \& Kurucz, R. L. 1997, A\&A, 318, 841

Casuso, E., \& Beckman, J. E. 2004, A\&A, 419, 181

Cignoni, M., Prada Moroni, P. G., \& Degl'Innocenti, S. 2003, MSAIS, 3,143

Grebel, E. K. 2004, in Origin and Evolution of the Elements: Carnegie Obs. Astrophys. Ser., 4, ed. A. McWilliams, \& M. Rauch (Cambridge: Cambridge Univ. Press)

Hurley, J., \& Tout, C. A. 1998, MNRAS, 300, 977

Ferrini, F., Matteucci, F., Pardi, M. C., \& Penco, U. 1992, ApJ, 387, 138

Kroupa, P. 2002, Science, 295, 82 [arXiv : astro-ph/0201098]

Mateo, M. 1998, ARA\&A, 36, 435

Pagel, B. E. J., \& Portinari, L. 1998, MNRAS, 298, 747

Pilyugin, L., \& Ferrini, F. 2000, A\&A, 354, 874

Pritzl, B. J., Knezek, P. M., Gallagher III, J. S., et al. 2003, ApJ, 596, L47

Quilis, V., Moore, B., \& Bower, R. 2000, Science, 288, 1617

Shore, S. N., \& Ferrini, F. 1995, Fund. Cosm. Phys., 16, 1

Skillman, E. D., Tolstoy, E., Cole, A. A., et al. 2003, ApJ, 596, 253

Skillman, E. D., Côté, S., \& Miller, B. W. 2003, AJ, 125, 593

Sollima, A, Ferraro, F. R., Pancino, E., \& Bellazzini, M. 2005, MNRAS, 357, 265

Tolstoy, E., Venn, K. A., Shetrone, M., et al. 2003, AJ, 125, 707

Tolstoy, E., Irwin, M. J., Helmi, A., et al. 2004, ApJ, 617, L119

Valle, G., Ferrini, F., Galli, D., \& Shore, S. N. 2002, ApJ, 566, 252

Valle, G., Shore, S. N., \& Galli, D. 2005, A\&A, 435, 551 (VSG05)

Vollmer, B., Cayatte, V., Balkowsky, C., \& Duschl, W. J. 2004, AJ, 561,708 